\title{
Évènements et travail de l'historien Le cas de la Révolution égyptienne de 1919 par Mohamed Sabri El Sorbunnî ${ }^{(1)\left(^{*}\right.}$
}

\author{
Dr. Amani Mostafa \\ Faculté des arts, Université du Caire
}

\section{Résumén:}

Cet article entend revisiter l'un des lieux de mémoire de l'histoire contemporaine de l’Égypte, celui de la Révolution de 1919, épisode qui a fait couler autant d'encre que de sang. Parmi les plumes qui se sont engagées à immortaliser les évènements de l'époque nous citons M. Sabri. Les deux volumes de La Révolution Égyptienne publiés successivement en 1919 et 1921, dressent un tableau, au dire de A. Aulard, des souffrances, des résultats et des espoirs de toute une nation et qui «ne sera lu nulle part avec plus d'intérêt et de sympathie que dans le pays qui a proclamé les droits de l'homme et les droits des peuples. ». Nous nous proposons donc d'examiner le travail de M. Sabri en tant qu'historien engagé, dans le but de dégager les procédés et techniques de l'écriture historique ainsi que les modalités et les schémas d'expression des évènements survenus en Égypte et qui lui ont prévalues le titre de « Révolution».

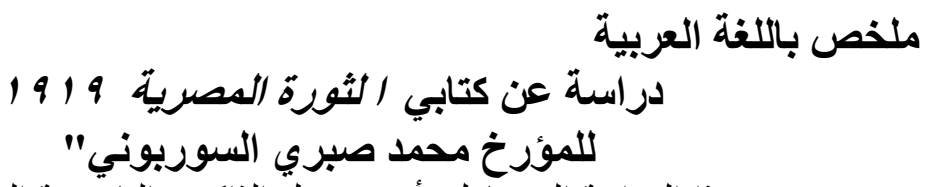

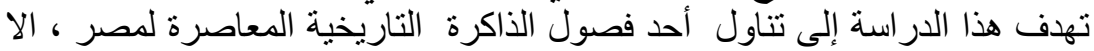

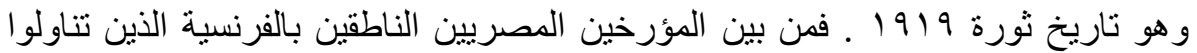

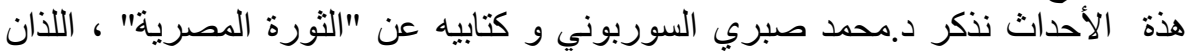

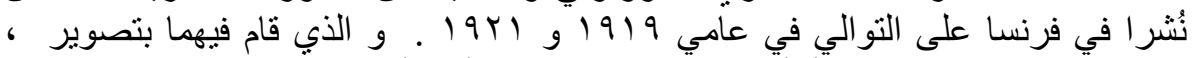

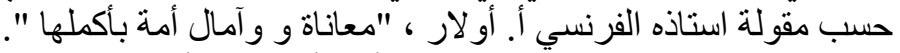

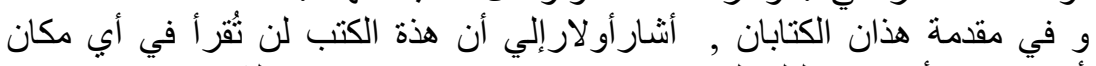

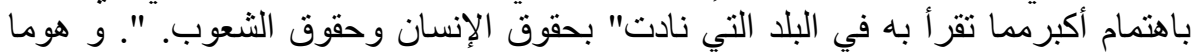

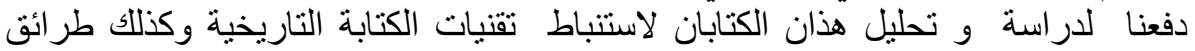

${ }^{(*)}$ Bulletin of the Faculty of Arts Volume 80 Issue 6 July 2020. 
و أنماط التعبير عن الأحداث التي وقعت في مصر و التي من شانها اقناع المجتمع الدولي

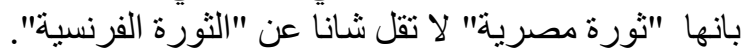

«De Lesseps, c'est le cas de le dire, profitera de son ascendant sur Saïd «Les vrais héritiers de Michelet, ce sont les historiens du Présent. (.....)Pascal peut-être avait raison : "Toute histoire qui n'est pas contemporaine est suspecte ${ }^{(2)}$ »

Cette étude se voudrait d'abord une tentative de définition de l'évènement dit révolutionnaire ainsi que les rapports qu'entretient l'historien vis-à-vis de l'évènement pour l'élaboration d'un récit historique.

M. Sabri, pour qui ne le connaît pas, est source d'inspiration pour tout chercheur dans le domaine de l'historiographie. Il est parti en France à ses frais en 1913 selon la volonté de son père et où il acquiert le surnom qui lui est très cher de «El Sorbunnî » ou «le sorbonnard ». Fameux collègue de Taha Hussein, il est cité à deux reprises dans son récit autobiographique la traversée intérieure ${ }^{(3)}$, ce qui laisse voir les traits d'un inlassable apprenti et d'un homme noble par nature.

Écrivain, poète, Docteur ès Lettres de l'université de Paris, Professeur à l'École Normale du Caire, fondateur et directeur de l'Institut des archives de l'université du Caire (1951); historien de l'art (le catalogue partiel et raisonné de sa bibliothèque privée sur les beaux-arts - art et histoire) en addition à ses commentaires de sa collection privée de peintures en sont la preuve la plus flagrante.

M. Sabri est renvoyé de l'Université lors des purges de 1952. Mais l'amertume d'une telle injustice ne l'a pas empêché d'offrir à Nasser son livre précieux les secrets de l'internationalisation du canal de suez et l'accord de 1888, publié 1957. La lettre de remerciement qui lui a été adressée de la part du président met en valeur le sérieux des recherches faites par l'historien. M. Sabri s'est éteint en 1978.

Revenons-en à la Révolution Égyptienne de 1919, texte d'expression française, écrit, par Mohamed Sabri, et préfacé par A. Aulard $^{(4)}$, chaire d'Histoire et grand historien de la Révolution 
française, est publié en France en deux tomes qui paraissent successivement en 1919-1921. Et où l'on voit notre historien brosser le tableau des souffrances et des espoirs des Égyptiens à l'heure même que leur Révolution s'est produite. La traduction du premier tome sur laquelle nous nous baserons en partie dans notre analyse, n'est offerte au public arabe que très tardivement en $2003^{(5)}$, quant à celle du deuxième, elle n'a pas encore vu le jour.

C'est dans un contexte politique difficile lié à l'occupation anglaise de l'Égypte, que M.Sabri a toujours eu à cœur de défendre la cause égyptienne et à faire connaître son vrai visage à l'opinion française. Devenant secrétaire de la délégation égyptienne à Paris, qui n'a rencontré de la part de la conférence de la paix qu' "une sourde Oreille et porte close ${ }^{(6)}$, il confia à Saad Zaghloul son espoir de voir s'écrire l'Histoire de L'Égypte selon les méthodes l'écriture historique contemporaine. "Si vous vous voulez que la révolution continue, avant l'arme et l'enthousiasme, il nous faut écrire l'Histoire de L'Égypte $^{(7)} \gg$ Ce qui poussa Saad Zaghloul à lui demander de remplir lui-même cette tâche ${ }^{(8)}$. Et M. Sabri ne tarde pas à faire de cette vocation l'unique mission de sa vie durant. Il publia en France, d'abord ses deux ouvrages sur la Révolution de 1919, puis sa thèse de Doctorat Genèse de l'esprit national de l'Égypte, Et cela avant de se lancer dans son projet qui a donné naissance à son chef-d'œuvre sur L'Empire égyptien sous Mohamed Ali suivi par L'Empire égyptien sous Ismaïl récemment traduit en Égypte en 2010.

En fait, Les deux tomes consacrés à la Révolution égyptienne de 1919, sont constitués au dire de notre historien, d'après des documents authentiques égyptiens, français, américains et même anglais. Les photographies prises au cours de la Révolution viennent étayer le discours historique et présentent à elles seules une innovation de la part de M. Sabri, puisque c'est tardivement dans les années 1930 que,

L'École des Annales appelait à un élargissement des sources documentaires parmi lesquelles la photographie et le cinéma devaient, tout naturellement, trouver leur place. Ce que réclamaient Marc Bloch et Lucien Febvre, ce que revendiqua, par la suite, la 
Nouvelle Histoire, dans son exhortation à l'étude de "nouveaux objets" (et qui) semble s'être, aujourd'hui, en partie, réalisé( ${ }^{(9)}$.

La parution de l'ouvrage de M. Sabri sur la Révolution Égyptienne de 1919 en France, devient donc une tentative de faire écouter la voix de l'Égypte. Une voix que les grandes puissances de l'époque ont travaillé à éteindre, étouffer voire soumettre. Ce qui fait, à vrai dire, de la publication de l'ouvrage à lui seul un Évènement. Donner pour titre à son ouvrage la Révolution égyptienne au moment où la presse française de masse s'accorde à nommer les évènements d'Égypte par les appellations d'"émeutes, de troubles et de désordre », annonce non seulement les couleurs de l'épopée révolutionnaire égyptienne mais aussi prévoit sa réussite.

Ce qui nous mène à souligner que le cas de $M$. Sabri est particulièrement celui de l'historien qui écrit l'histoire vivante de son temps, en tant qu' « explorateur de l'actuel » pour garder l'expression de Marc Bloch.

Certes l'explorateur de l'actuel et celui des époques lointaines ont chacun leur façon particulière de manier l'outil. Chacun aussi, selon le cas, possède l'avantage. Le premier touche la vie d'une prise plus immédiatement sensible; le second, dans ses fouilles, dispose de moyens qui sont souvent refusés au premier. Ainsi la dissection du cadavre, en découvrant au biologiste bien des secrets que l'étude du vivant lui aurait laissés ignorer, se tait sur beaucoup d'autres, dont le corps vivant seul détient la révélation ${ }^{(10)}$.

Et c'est à ce propos, que le travail de l'historien, basé sur l'observation, réside surtout dans la sélection des évènements dignes d'être présents dans son récit historique. Puisque "l'on sait, aujourd'hui, que toute histoire est écriture, narration, mise en intrigue: c'est par le récit que l'historien organise et structure les faits et évènements du passé et qu'il leur donne un sens ${ }^{(11)}$."

\section{Qu'est-ce qu'un évènement révolutionnaire ?}

Or, pour définir un évènement révolutionnaire dans un contexte imprégné par le discours colonialiste occidental, l'on peut croire qu'il est d'abord conçu comme un changement brusque, inattendu, faisant 
rupture avec ce qui précède et préparant peut être à un bouleversement de situation sociale et politique. L'évènement devient ainsi, selon l'expression de François Furet, « une sorte de point focal où vient s'abolir le passé, se constituer le présent et se dessiner l'avenir ${ }^{(12)}$. » Lesquels donc des évènements survenus en Égypte méritent de figurer dans le récit de $\mathrm{M}$. Sabry et quels sont les modalités utilisées par l'historien pour les faire connaître?

\section{Faire connaître l'évènement}

Partant de la thèse de Pierre Nora dans son «évènement monstre » selon laquelle «pour qu'il y ait évènement il faut qu'il soit connu $^{(13)} »$. Nous constatons que M. Sabri, dès le premier volume de son livre, a travaillé à «faire connaître » la Révolution égyptienne à l'opinion française, qu'elle est surtout en droit fil de sa Révolution, qu'elle partage soit ses valeurs soit ses symboles. Par le drapeau mariant la croix et le croissant le lectorat français retrouve l'emblème de la Révolution de 1789 réconciliant «la bannière de Paris à l'oriflamme royale ${ }^{(14)} »$. Les crimes commis par les Anglais sont comparés à ceux des Turcs contre les Grecs de Chio et trouvent leurs représentations dans le titre «Après les Turcs, les Anglais ont passé par là ». Titre qui revoit bien entendu au poème célèbre de Victor Hugo intitulé $L$ 'enfant ${ }^{(15)}$. L'exil et l'enfermement sans jugement sont les supplices subis par des centaines de nationalistes Égyptiens. Pour le moindre mot prononcé contre les Anglais, les Égyptiens deviennent les hôtes de «Liman Torah », cette prison à proximité du Caire, où flagellations et «Kourbag » sont d'usage récurrent. Elle rappelle donc aux Français « la Bastille», symbole de l'absolutisme royal depuis Richelieu. Et finalement, «La Marseillaise d'un peuple opprimé » est le titre que donne M. Sabri à sa traduction des vers d'un poème de trois quatrains chanté par les Égyptiens dans les rues et qui présente l'image de l'Égypte souriante devant la mort ${ }^{(16)}$.

Quant au second tome, nous nous arrêterons d'abord devant l'évènement inaugural ainsi que celui de clôture et entre ces deux volets, nous nous intéresserons au rapport que tient l'écriture de l'évènement révolutionnaire à la photographie. 


\section{L'évènement inaugural du récit historique / Un Entretien fait Événement}

À première vue, nous constatons qu'au titre de l'ouvrage viennent s'ajouter les sous - titres des chapitres à savoir « La révolution en marche - les origines du Boycottage de la mission Milner- Le Rameau d'olivier de Lord Milner- Dans le dédale du boycottage- La Révolution dans les âmes - L’Esprit de la Révolution et l'œuvre de la délégation Le projet Milner », dont le rôle est de présenter au regard des lecteurs français la Révolution égyptienne de 1919 dans sa période de réalisation.

Quant à la mise en récit de l'évènement en histoire, Arlette Farge dit :

Le récit de l'événement est sa pierre angulaire; mais sous ce vocable on peut entendre énormément de choses extrêmement différentes. Sur le lien entre histoire et événement, beaucoup a déjà été dit et la discipline s'est toujours posé des questions à cet égard. La nature et l'essence de l'événement, le bien-fondé de son choix parmi d'autres pour en faire une analyse significative, la place, porteuse de conséquences, de son accession au récit historique ont été l'objet de maintes discussions, selon les époques et aussi les écoles de pensée ${ }^{(17)}$.

Notre historien pour ainsi dire, choisit de commencer son récit historique du second volume par ce qu'il considère comme «un évènement mémoriel ». Il s'agit de la prise de parole par les grands leaders Égyptiens avec à leur tête Saad Zaghloul, dans un entretien datant du 13 novembre 1919 et Sir Wingate Pacha pour lui communiquer la volonté d'indépendance complète d'Égypte. Cet entretien vient Suite à la déclaration unilatérale du protectorat et son régime provisoire instauré pendant la guerre.

C'est dans cet entretien que l'on voit un condensé des lieux communs du discours colonialiste de l'époque. Nous en citons quelques exemples :

«Les Égyptiens n'ont pas d'opinion publique prévoyante», "pour un enfant (qu'est l'Égypte) trop de nourriture est cause 
d'indigestion », "La proportion des illettrés dans la population égyptienne est plus forte qu'ailleurs », "L'Égypte était l'esclave de la Turquie. En Étant l'esclave de l'Angleterre, serait-elle dans une position inférieure?", "La situation géographique et militaire de l'Égypte l'expose au danger de tomber sous la domination de toute nation forte autre l'Angleterre ${ }^{(18)}$. »

Selon Sabri, les Réponses avancées par Saad Zaghloul et ses collègues à Wingate pacha et qui relèvent d'un programme défini revendiquant courageusement l'indépendance complète de l'Égypte, semblent «poser la première pierre au monument de l'indépendance » au point de fêter ce jour comme «IndépendanceDay » jour dont l'anniversaire est fêté dans toute l'Égypte mais qui a cessé de l'être à présent. Raison pour laquelle M.Sabri l'a choisi comme «évènement inaugural » du deuxième tome.

\section{Évènement révolutionnaire et photographie militante :}

Si l'on revient au rapport évènement /photographie, nous constatons que la triple fonction des photographies authentiques dans le texte en question est de montrer premièrement les crimes commis par les Anglais contre la population égyptienne: flagellations, fusillades et violentes représailles.

Deuxièmes, de souligner et d'affirmer l'engagement de toutes les couches sociales,

«(Les fellahs) marchèrent la main dans la main, avec les juges, les magistrats, les étudiants, les ulémas, les prêtres et les ouvriers, réalisant ainsi l'union sacrée de tout un peuple [] Dans les grandioses funérailles nationales des morts pour la patrie » ${ }^{(19)}$.

Et dernièrement de faire "voir [] S'écrouler les deux grands préjugés séculaires de religion et de sexe, qui ont été pour l'humanité à l'origine de tant de guerres et de tant de discordes. Préjugé de religion: on voyait les musulmans entrer dans les églises et les Coptes dans les mosquées prêcher le culte de la patrie. Le drapeau égyptien symbole de cette unité, portait la croix et le croissant. Préjugé de sexe : on voyait les femmes descendre en masse sur la place publique, 
pénétrer dans l'arène politique, discourir et inciter les hommes, par le geste et par la parole, au plus haut patriotisme ${ }^{(20)}$.

La multiplication du verbe «voir» a pour effet de croiser le textuel au visuel de façon à faire des photographies un document historique essentiel et indispensable à la défense de la cause égyptienne.

L'engagement des Égyptiennes de toutes les classes sociales se trouve au cœur des événements fondamentaux de la Révolution de 1919.

Le premier (spectacle) était des femmes de la haute société montées dans des voitures et des automobiles. La plupart portaient des drapeaux égyptiens ou des rubans plus patriotiques. De nombreuses mères habillaient leurs enfants du drapeau égyptien. (...) Sans cesse, elles se penchèrent hors des voitures et donnèrent le signal au hurlement antiphonaire de la foule. (...) c'est l'Orient, la vraie citadelle de la séquestration et de la subordination des femmes. Ce grand jour, cependant, amena les femmes de toutes les classes à l'avant comme incitatrices et comme chefs des hommes. Cela seul donna à l'occasion un caractère révolutionnaire » (21)

Le patriotisme féminin est exalté dans le domaine public pour mettre en valeur les actions de femmes réelles. Elles sont pour ainsi dire présentes dans les représentations des événements déterminants comme les manifestations du 9 avril 1919 et décrites comme des héroïnes ayant sacrifié leurs vies pour la cause d'indépendance de leur pays. «Un long cortège d'Égyptiennes se déroule dans les rues du Caire, les troupes anglaises lui barrent le passage, et le dispersent... Tuez - moi pour qu'il y ait une miss Cavell en Égypte ! Jette une de ces héroïnes à un officier qui la brutalise ${ }^{(22)}$. »

\section{Événement et discours de la presse occidentale}

Quant à L'évènement qui vient clôturer le récit historique, il s'agit du «projet Milner », accord au premier lieu présenté, du côté anglais, comme un aboutissement d'une Révolution égyptienne réussie. Cet accord jouit d'une importance particulière car l'historien a choisi de le placer dans son contexte médiatique de la presse anglaise, 
française et américaine. Le dernier chapitre du deuxième tome, n'est en effet, qu'un état des lieux des partis pris de la presse occidentale de masse vis-à-vis de ce projet. Pour ne garder que l'opinion française nous citons les journaux : Liberté, Écho de Paris, L'intransigeant, Journal des Débats, l'Avenir, Libre parole. C'est à cet endroit que M. Sabri multiplie les critiques du projet d'accord pour l'indépendance de l'Égypte de façon à étayer sa thèse sur un projet d'indépendance fallacieux et voué à l'échec et cela après avoir conclu que :

Tout projet qui ne ferait que continuer le protectorat sera un défi perpétuel à tout un peuple, la consommation définitive d'un crime prémédité depuis un siècle, la violation des soixante engagements qui lient l'honneur même de l'Angleterre, la consécration du triomphe de l'impérialisme britannique dans tout l'Orient et la raison d'être d'une Révolution perpétuelle. L'Égypte hérö̈que et immortelle, fière de son passé, confiante en son avenir, persévérante et laborieuse, ne saura souscrire qu'à son indépendance complète ${ }^{(23)}$.

\section{Conclusion}

Nous concluons que M.Sabri, par son travail d'historien, a bien réussi de montrer que la Révolution égyptienne de 1919 n'est pas un évènement sans causes. L'état de l'Égypte à la veille de la Révolution se lit dans un tableau préalable celui de l'impérialisme occidental. Il a pu fixer par son recours aux photographies authentiques, le canevas des évènements révolutionnaires tout en gardant de vue, la devise de la Révolution française de 1789 : vivre libre ou mourir.

«Voilà donc l'historien appelé à rendre ses comptes. Il ne s'y hasardera qu'avec un peu de tremblements intérieurs : quel artisan, vieilli dans le métier, s'est jamais demandé, sans un pincement de coeur, s'il a fait de sa vie un sage emploi ? ${ }^{(24)}$. » 
Figure (1)

\section{LETTRE-PREFACE}

$$
\text { Paris. } 25 \text { sowere toty. }
$$

\footnotetext{
Won cher Sabry.

1. ivens ale lire les iprenzes de zotre lize

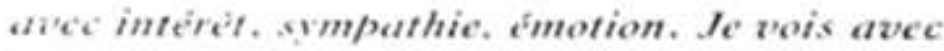
platis ghe mime guand zous wous faites I avowat ate zotre chire patric. metme quand wotre catur suffre, zous n'oubliez pas les lecons ac mëthole historigue que zous are emportées de la Sorfonne, oì vous azez efte un étudiant si zcké et si aimable. Vous zous etes alocumenté whe sin, et wous n'aze $=$ rien affirme que sur pieces. Iotre ricit mous touche et hous remue, thon sulement par une éloquence de patriote, thais par un air et comme une odeur de vérite.

It ne connatis pas seulement I'Egypte par Ithisfoire. ot ce riest pas seulement par les liares unc ïai appris à révérer la noblesse de
} 
Figure (2)

viII

1.ITTKE-PRËFACE.

votre peuple. qui fut un des initiateurs de la raison humaine. I'ai voyagé dans ce merveilleux pays, je the suis baigné le corps et l'esprit dans rotre lumiere, jai vu les expressives fiqures de wos compatriotes, $j$ ai vu ces lignes yracicuses et ces vils visages fins, dont la frappante ressemblance avec les anciens Eyyptichs sculptés sur vos monuments atteste la purete de votre race (et quelle autre s'est conseruíe aussi pure?), enfin j'ai bu de l'eau du Nil: c'est wous dire que IÉgypte ne m'est pas étrangère et que je l'aime.

1/ais je ne l'aime pas seulement comme on aime un musée, et pour ses nécropoles. C'est une terre de vivants. Votre renaissance est admirable, renaissance intellectuelle, renaissance économique. Vous avez besoin de la liberté pour développer votre génie et votre avenir. Je sais que vous en êtes dignes. Nos étudiants égyptiens, à la Sorbonne, ont fait paraitre un amour viril de la vérité. Président de la Mission laïque française à l'époque où je voyageais chez vous, j'ai vu combien les Egyptiens aiment les lumieres, et en particulier la culture francaise. Vous voulez vivre comme peuple, et vous invoguez les principes de notre 
Figure (3)

Révolutign, qui en effet se distingue d'autres en ce qu'elle ria has dre faite pour nous seuls, mais pour hous lès hommes. Vous comptez, pour avoir te droit de vivre votre vie, sur la Société des nafions, gui niest pas seulement fille de la pensée americaine, mais de la pensée franfaise. Vous avez bien raison et votre énergie, si heroïguement dévouée (avec un sens délicat de la mesure), fera votre destinde. Parmi tant de peuples que cette guerre a ressuscites et qui lèvent la piòre de leur tombeau, la figure du peuple égyptien est resplendissante d'avenir.

Peu a peu, ropinion, non seulement franfaise, mais mondiale, se rallie d potre cause. Les belles pages que vous venez d'ecrire vont encore hater ce ralliement, wont Puniversaliser, et je prédis un entrainement de sympathie, qui, si vous bersoburex dans cette heroĩgue obstination a viond fomne peuple, gagnera iusqu'au peuple anplais Tiri-meme: I'histoire nous apprend qu'th ta jamais resiste itrdefniment à la raison af a la justice.

L'Egyptel be he ia wois pas seulement dans son vieux passe splendide: je la vois dans son avenir, belle, forte, utile ì Thumakith. 
Dr.Amani Mostafa : Évènements et travail de l'historien

Figure (4)

le vous remercie, mon cher Sabry, de m'avoir donné, par votre live, cette espérance et cette vision. Wive notre noble peuple!

$$
\text { A. AULARD, }
$$

professeur d'histoine de la Refolution française

a l'Universile de Paris. 


\section{Notes :}

(1) M. Sabri (1890-1978). Dès son plus jeune âge, ses études renforcent son goût pour la poésie qui occupe une place de choix dans la vie de l'historien, nous nous référons à cet égard à la liste de ses ouvrages écrits en arabe et diffusé sur le site de la bibliothèque centrale de l'université du Caire. Il occupa plusieurs Postes : enseignant à l'École normale (1924), à l'Université du Caire (1925), à l'École de langue arabe (1927) ; directeur de la Mission égyptienne à Genève (1934-1937); des Éditions égyptiennes (1938); vice-directeur de la Bibliothèque nationale; suite au rejet de sa promotion, il intente un procès à l'État et démissionne. Iman Farag «Les manuels d'histoire égyptiens ». Genèse et imposition d'une norme ». Genèses 2001/3 (no44), p.4-29. DOI 10.3917/gen.044.00044

(2) Pierre Nora, «L'événement monstre». In: Communications, 18, 1972.

L'événement. pp. 162-172;doi : 10.3406/comm.1972.1272

http://www.persee.fr/doc/comm_0588-8018_1972_num_18_1_1272

(3) «Le second de ces Égyptiens téméraires n'était que le docteur Sabri el Surbunnî. Il s'attela à la tâche avec beaucoup d'énergie se présenta plusieurs fois, mais il fut arrêté par l'obstacle du latin. Le jour de l'examen, quand il découvre le texte de la version latine, il n'y jeta qu'un rapide coup d'œil, puis le plia en quatre et rendit copie blanche. Il quitta ensuite la salle en riant et en déclamant quelques vers fameux, empruntés à un poète classique, et exprimant le désespoir. Il n'était pas désespéré le moins du monde, ni résigné devant la difficulté. Il redoubla au contraire d'efforts et, quelques années plus tard, fut en mesure de traduire son texte, ce qui lui valut de devenir licencié ès Lettres. », p.152 puis «Le succès fut pourtant au rendez -vous. Ce fut le docteur Sabri el Sorbunnî qui lui apporta la bonne nouvelle. Il vint chez notre ami un soir, au comble de la joie et à bout de souffle pour avoir couru tout au long du chemin, depuis la Sorbonne et pour avoir grimpe les escaliers quatre à quatre jusqu'au sixième étage. Dès qu'on lui ouvrit la porte, il s'empressa d'annoncer la nouvelle puis tourna les talons et repartit, aussi vite qu'il était venu, sans même songer à se reposer un instant. Cette année- la, cet excellent collègue s'était lui-même présenté à l'examen mais avait rendu feuille blanche en version latine, après avoir pris connaissance du texte ; puis il s'était éclipsé en riant et en déclamant quelques vers désespérés, comme nous l'avons raconté plus haut. Il était vraiment surprenant de le voir, à présent, rayonnant de joie, heureux pour son jeune ami au point d'en oublier le sentiment de son propre échec. » Taha Hussein, la traversée intérieure, Ed. Gallimard, Paris, 1992, pp.154-155

(4) Nous reproduisons dans l'annexe de cette étude la lettre-préface de A. Aulard présente dans la version incomplète du premier tome d'expression française, et 
qui nous a été fourni par Mme Mona Mohamed Sabri, la fille de l'historien. Figure 1,2,3 et 4 .

(5) Il s'agit de la traduction du premier tome de l'ouvrage, parue en 2003, dans le cadre du projet national de traduction par Magdy abd el Hafez et Ali Kurhan. Elle est le fruit d'une trouvaille faite par Caroline Kurhan qui apporta de la bibliothèque de l'ile de Corse, une copie d'expression française, presque unique en Égypte, à notre connaissance, du premier tome de l'ouvrage de M.Sabri sur la Révolution de 1919. Muhammad Sabri, La Révolution égyptienne, Tome I, traduction de Magdi abd el Hafez et Ali Kurhan, éd. du haut conseil de culture, le Caire, 2003. P.9

(6) Je me permets de citer mon article intitulé, « La voix de L'Egypte, voix étouffée Vs voix prêtée, publié in actes du colloque de l'Association Égyptienne des professeurs de français, Francophonie arabe : voix et voies, 67 avril 2017, Bibliotheca Alexandrina, éd. Horizons, Numéro spécial, 2017, pp.344-353

(7) Il est connu qu'avant M.Sabri, l'Histoire de l'Égypte est écrite par des européens, nous citons en l'occurrence Histoire de la Nation Égyptienne (1931), écrite par Hanotaux sous la commande du roi Fouad.

(8) Muhammad Sabri, Genèse de l'esprit national d'Egypte (1863-1882), traduction de Nagui Ramadan Attia, éditions Dar al-Kûtûb wa al-wathayiq al qawmia, le Caire, 2011.

(9) Ilsen About et Clément Chéroux, « l'histoire par la photographie », Etudes photographiques, 10 novembre 2001, [en ligne], mis en ligne le 18 novembre 2002. URL ; http//études photographiques. Revues.org/261.consulte le 27 Janvier 2017.

(10) Marc Bloch (1886-1944), Apologie pour l'Histoire ou métier d'Historien, Cahier des Annales, 3, Librairie Armand Colin, Paris, $2^{\mathrm{e}}$ édition, 1952, p.34

(11) Richard Jacquemond, écrire l'Histoire de son temps (Europe et monde arabe), L'écriture de l'Histoire I, Etudes transnationales, francophones et comparées, éditions L'Harmattan, Paris, 2005, p.7

(12) François Furet, Penser la Révolution française, collection Folio/Histoire, éd. Gallimard, 1978, p.40

(13) Pierre Nora, «l'évènement monstre », l'article cité, p.162

(14) M.sabri citant Victor Marguerite, La Révolution égyptienne, Tome I, traduction de Magdi abd el Hafez et Ali Kurhan, op.cit., p.51

(15) «Les Turcs ont passé là. Tout est ruine et deuil. », Ce poème en addition au tableau de De Lacroix Les massacres de Scio, sont deux manifestes du Romantisme français du début du XIX ${ }^{\text {ème }}$ siècle, immortalisant les scènes de répressions violentes exercées contre les Grecs de l'Ile de Chio, Victor Hugo, Les Orientales, « l'enfant », éd. Charles Gosselin, tome III, Paris, 1829, p.187

(16) M. Sabri, La Révolution égyptienne, Tome I, traduction de Magdi abd el Hafez et Ali Kurhan, op.cit., p.60 
(17) Arlette Farge, "Penser et définir l'événement en histoire", Terrain, numero-38 Qu'est-ce qu'un événement? (mars 2002), [En ligne], mis en ligne le 06 mars 2007. URL : http://terrain.revues.org/index1929.htm. Consulté le 24 janvier 2009.

(18) M. Sabri , La Révolution égyptienne, Tome II, éd. Librairie J. Vrin, Paris, 1921, p. $10-14$

(19) M. Sabri, La Révolution égyptienne, Tome II, op.cit., p.24.

(20) Ibid, p.22-23.

(21) M. Sabri, La Révolution égyptienne, Tome I, version française incomplète, sans date ni édition, p.74

(22) M. Sabri, La Révolution égyptienne, Tome I, traduction de Magdi abd el Hafez et Ali Kurhan, op.cit., p.52 scène citée par Victor Marguerite dans son livre, La voix de l'Égypte, Librairie Plon, Paris, 1919, p. 13 et reprise par M.sabri.

(23) M. Sabri, La Révolution égyptienne, Tome II, op.cit., p.246.

(24) Marc Bloch (1886-1944), Apologie pour l'Histoire ou métier d'Historien, op.cit., p.60. 


\section{Corpus}

\section{Bibliographie}

Muhammad Sabri, La Révolution égyptienne, Tome I, version incomplète, sans date ni édition.

Muhammad Sabri, La Révolution égyptienne, Tome I, traduction de Magdi abd el Hafez et Ali Kurhan, éd. du haut conseil de culture, Le Caire, 2003.

Muhammad Sabri, La Révolution égyptienne, Tome II, éd. Librairie J. Vrin, Paris, 1921.

\section{Ouvrages de Références}

François Furet, Penser la Révolution française, collection Folio/Histoire, éd. Gallimard, 1978.

Marc Bloch (1886-1944), Apologie pour l'Histoire ou métier d'Historien, Cahier des Annales, 3, Librairie Armand Colin, Paris, $2^{\mathrm{e}}$ édition, 1952.

Muhammad Sabri, Genèse de l'esprit national d'Egypte (1863-1882), traduction de Nagui Ramadan Attia, éditions Dar al-Kûtûb wa al-wathayiq al qawmia, Le Caire, 2011.

Taha Hussein, Al-Ayyam (titre original), La traversée intérieure, traduit de l'arabe par Guy Rocheblave, préface d'Etiemble, éditions Gallimard, Paris, 1992.

Victor Marguerite, La voix de l'Égypte, Librairie Plon, Paris, 1919.

\section{Actes de colloques}

Amani Mostafa," La voix de L’Égypte voix étouffée vs voix prêtée", publié in actes du colloque de l'Association Égyptienne des professeurs de français, Francophonie arabe: voix et voies, 6 au 7 avril 2017, Bibliotheca Alexandrina, éd. Horizons, Numéro spécial, 2017, pp.344-353

Richard Jacquemond, écrire l'Histoire de son temps (Europe et monde arabe), L'écriture de l'Histoire, Études transnationales, francophones et comparées, éditions L'Harmattan, Paris, 2005. 


\section{Articles}

Arlette Farge, "Penser et définir l'événement en histoire", Terrain, numero-38 - Qu'est-ce qu'un événement? (mars 2002), [En ligne], mis en ligne le 6 mars 2007. URL : http://terrain.revues.org/index1929.htm. Consulté le 24 janvier 2009.

Ilsen About et Clément Chéroux, «l'histoire par la photographie », Etudes photographiques, 10 novembre 2001, [en ligne], mis en ligne le 18 novembre 2002. URL; http//études photographiques. Revues.org/261.consulte le 27 janvier 2017.

Iman Farag « Les manuels d'histoire égyptiens. Genèse et imposition d'une norme ». Genèses, 2001/3 (no 44), p. 4-29. DOI 10.3917/gen.044.00044

Pierre Nora, "L'événement monstre ». In : Communications, 18, 1972. L'événement. pp. 162-172 ; doi : 10.3406/comm.1972.1272 http://www.persee.fr/doc/comm_0588-8018_1972_num_18_1_1272 